\title{
"Unlocking" the Ground: Increasing the Detectability of Buried Objects by Depositing Passive Superstrates
}

\author{
Constantinos A. Valagiannopoulos, Member, IEEE, Nikolaos L. Tsitsas, Member, IEEE, \\ and Ari H. Sihvola, Fellow, IEEE
}

\begin{abstract}
One of the main problems when trying to detect the position and other characteristics of a small inclusion into lossy earth via external measurements is the inclusion's poor scattering response due to attenuation. Hence, increasing the scattered power generated by the inclusion by using not an active but a passive material is of great interest. To this direction, we examine, in this work, a procedure of "unlocking" the ground by depositing a thin passive layer of conventional material atop of it. The first step is to significantly enhance the transmission into a lossy half space, in the absence of the inclusion, by covering it with a passive slab. The redistribution of the fields into the slab and the infinite half space, due to the interplay of waves between the interfaces, makes possible to determine the thickness and permittivity of an optimal layer. The full boundary value problem (including the inclusion and the deposited superstrate) is solved semi-analytically via integral equations techniques. Then, the scattered power of the buried inclusion is compared to the corresponding quantity when no additional layer is present. We report substantial improvement in the detectability of the inclusion for several types of ground and burying depths by using conventional realizable passive materials. Implementation aspects in potential applications as well as possible future generalizations are also discussed. The developed technique may constitute an effective "configuration (structural) preprocessing" which may be used as a first step in the analysis of related problems before the application of an inverse scattering algorithm concerning the efficient processing of the scattering data.
\end{abstract}

Index Terms-buried inclusion, inverse scattering, integral equations, detectability, mixing formulas

\section{INTRODUCTION}

$\mathbf{F}$ INDING a buried object inside the earth is a very interesting problem with long history. It is motivated by important applications including detection of mines [1], [2], mineral deposits [3], [4], and unexploded ordnances (UXOs) [5], [6] as well as scattering by buried pipes [7], [8], model trees [9] and surface and subsurface inhomogeneities (e.g. rocks) [10].

Various methods have been established for the localization and reconstruction of such objects. Characteristic representatives are methods employing primary fields generated by ground-penetrating radars (GPRs) using waves from a moving

C. A. Valagiannopoulos is with the Department of Physics, Nazarbayev University, Astana 010000, Kazakhstan (e-mail: konstantinos.valagiannopoulos@nu.edu.kz).

N. L. Tsitsas is with the Department of Informatics, Aristotle University of Thessaloniki, Thessaloniki 54124, Greece (e-mail: ntsitsas@csd.auth.gr).

A. H. Sihvola is with the Department of Radio Science and Engineering, Aalto University, Aalto 00076, Finland (e-mail: ari.sihvola@aalto.fi).

Manuscript received December 10, 2015; revised January 24, 2016. radar, lying in close proximity to the ground, in order to scan and image the subsurface by recording the strength of the echo produced by the interaction between the impinging waves and the buried objects received at the air-soil interface in a (usually) multi-static configuration. GPRs allow non invasive diagnostics of the probed domain in a fast and simple way and are widely employed in civil engineering, shallow subsurface prospecting applications and archaeology. Representative techniques as well as application domains are reviewed in [11] and [12]. Important characteristics of GPR methods are the number of utilized frequencies as well as the type of the primary field (pulse or harmonic wave). More precisely, harmonic primary fields are employed in combination with single-frequency measurements in [13]-[17] and with multiplefrequency ones in [12] and [18]-[20]. Moreover, GPR pulses (obviously possessing continuous spectrum) are alternatively considered as primary excitations in several studies implementing GPR techniques (indicatively, we cite [21]-[24]). All of the above investigations [13]-[24] utilize multiple near-field receivers. Besides, we note that plane waves have been also employed as primary fields for the detection and localization of buried objects (see e.g. [10], [25], [26] and [27]).

Most of the above mentioned methods rely mainly on the establishment of improved algorithms concerning the processing of the scattering data in order to make the location of the buried object more apparent. In this work, we establish and implement a different approach which aims to increase the detectability of a buried inclusion and, in this way, prepare the ground for a more effective implementation of such algorithms. In particular, we modify the structure of the considered configuration by depositing on the lossy earth a suitable thin reciprocal and passive superstrate layer which assists both the primary (line-source) field's penetration in the lossy earth as well as the buried inclusion's scattering response in the air region. Our main objective is to determine the proper permittivity and electric thickness of the superstrate in order to increase (make more feasible) the detectability of a cylindrical perfectly electric conducting (PEC) inclusion located at a certain distance from the surface of the ground.

We solve semi analytically the scattering problem via integral equation techniques. More precisely, the associated boundary value problem is reformulated via a Fredholm integral equation for the current flowing on the surface of the PEC inclusion (see also the discussions in [28]). This integral equation is subsequently solved by a semi-analytical methodology 
providing high numerical stability and controllable accuracy. Then, we show that the choice of our optimal superstrate layer is not significantly affected by the size and depth of the inclusion and therefore it is actually the inclusion-free configuration that primarily dictates our choice.

Wet, medium dry as well as very dry types of ground are considered and for each of these types specific realizable mixtures of ordinary/natural materials are reported, which accomplish the specific objectives of making the presence of the inclusion more visible, by maximizing the magnitude of transmitted wave into the lossy ground. It is worth to emphasize that we consider a passive superstrate layer in order to amplify the scattering response of the buried inclusion. We use a harmonic GPR primary field and utilize single-frequency far-field measurements. We present several numerical results demonstrating that it is indeed possible to amplify significantly the scattering response of the inclusion by covering the ground with a suitable superstrate layer.

We note that the idea of modifying the effective properties of the ground in order to reduce background medium loss and provide improved detection of a buried target has been investigated to some extent in [29]-[31]. More precisely, in [29] a simple physical optics model was utilized for the analysis while modifications of the ground by adding a large quantity of water along with an amount of liquid nitrogen were proposed. In [30] it was shown experimentally that an artificial dielectric, composed of an array of small insulated metalcoated plastic spheres and lossless uniform plastic spheres, can be placed over a chosen area and mitigate clutter effects due to ground surface roughness. The improved detection of a mine after covering a rough surface with a smoothed layer of appropriate sand was experimentally demonstrated in [31].

The developed technique in the present work actually constitutes an effective "configuration (structural) preprocessing" which may be used as a first step in the analysis of related problems. Practical limitations like the presence of noise and clutter as well as measurement sensitivity aspects are expected to be treated/remedied by algorithmic procedures which will follow as next steps based on already existing processing methods (like e.g. the ones of [13]-[24]). The next step towards the practical implementation of the presented approach is to test how the existing algorithms cooperate with our method and evaluate possible weaknesses originating from the change of the configuration by adding the superstrate on the ground.

An $\exp (+j 2 \pi f t)$ time dependence is assumed and suppressed throughout the analysis, where $f$ is the operational frequency of the single-frequency radar.

\section{Boundary Value Problem}

The two dimensional (2-D) geometrical configuration of the boundary value problem under consideration is depicted in Fig. 1(a). The utilized Cartesian coordinate system $(x, y, z)$ is also shown; the corresponding cylindrical coordinate system $(\rho, \phi, z)$ can be used interchangeably. A cylindrical inclusion of circular shape (with radius $a$ ) and perfectly electric conducting (PEC) boundary is located at distance $d$ into a half space filled with a medium of relative complex permittivity $\varepsilon_{r 1}$. This half space is assumed to model the earth while the buried PEC cylinder is considered electrically small, i.e. $k_{0} a \ll 1$, where $k_{0}=\frac{2 \pi f}{c}$ is the free-space wavenumber with $c=\frac{1}{\sqrt{\varepsilon_{0} \mu_{0}}}$ the speed of light (in vacuum with permittivity $\varepsilon_{0}$ and permeability $\left.\mu_{0}\right)$. The objective of this study is to select the thickness

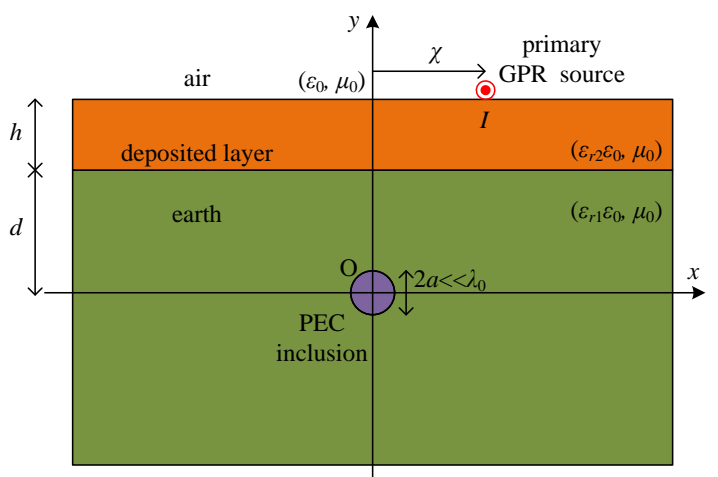

(a)

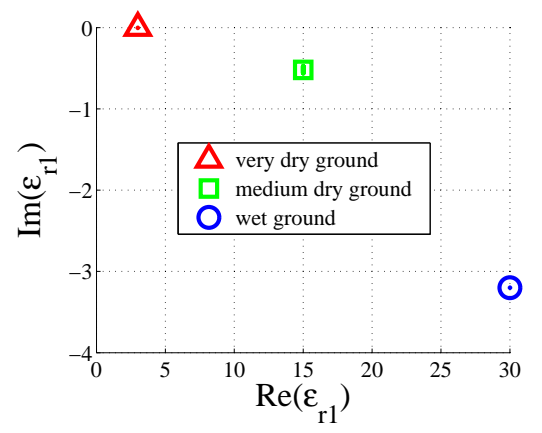

(b)

Fig. 1. (a) Geometrical configuration of the structure under investigation: we deposit a dielectric layer of suitable characteristics (height $h$, permittivity $\varepsilon_{r 2}$ ) on the host half space (permittivity $\varepsilon_{r 1}$ ) to increase the detectability of a buried inclusion at depth $d$. The inclusion is considered as perfectly electrically conducting (PEC), has electrically small size $2 a$ ans is excited by an electric line source of current $I$ positioned on the vacuum-superstrate surface at horizontal distance $\chi$ from the inclusion. (b) The dielectric constant $\varepsilon_{r 1}$ represented on its own complex plane when the host medium is the earth for three different types of ground at UHF frequency range.

$h$ and the (possibly complex) relative permittivity $\varepsilon_{r 2}$ of an electrically thin layer $\left(k_{0} h<1\right)$, deposited atop the earth, in order for the detection of the inclusion's location to become more feasible. The primary field is due to a moving line source carrying electric current $I$ and located at $(x, y)=(\chi, d+h)$; its vertical position is fixed at $y=d+h$ while its horizontal position $x=\chi$ may vary. Such a primary excitation is expected to model practical implementations related to GPRs. The entire space has constant permeability $\mu_{0}$.

The configuration is illuminated by a $z$-polarized primary electric field due to a line source in free space (region $\# 0$ ). The field's $z$-component is given by the Fourier integral [26]

$$
\begin{aligned}
E_{0, i n c}(x, y)= & -\frac{j I k_{0} \zeta_{0}}{4 \pi} \times \\
& \int_{-\infty}^{+\infty} \frac{e^{-g_{0}(\beta)|y-d-h|}}{g_{0}(\beta)} e^{-j \beta(x-\chi)} d \beta,
\end{aligned}
$$


where $\zeta_{0}=\sqrt{\frac{\mu_{0}}{\varepsilon_{0}}}$ is the free-space wave impedance and $g_{0}(\beta)=\sqrt{\beta^{2}-k_{0}^{2}}$ is evaluated with positive real part and, in case it is zero, with positive imaginary part.

Due to the 2-D nature of the configuration and the incident field, all the generated electric fields in each region will be also $z$-polarized and described in the sequel by their $z$-components.

The formulation of an integral representation of the scattered field by the configuration of Fig. 1(a) requires suitable analytic expressions of (i) the fields induced on the homogeneous (without the PEC cylinder) structure, due to the primary cylindrical wave (1), and (ii) the Green's function of the homogeneous structure.

First, we solve the associated boundary value problem, in the absence of the cylindrical inclusion, and find that the $z$-component of the background electric field into the host medium (region \#1) takes the form

$$
E_{1, b a c k}(x, y)=\int_{-\infty}^{+\infty} \gamma_{b a c k}(\beta) e^{g_{1}(\beta) y} e^{-j \beta(x-\chi)} d \beta,
$$

where

$$
\begin{aligned}
& \gamma_{\text {back }}(\beta)=\frac{j I k_{0} \zeta_{0}}{\pi} g_{2}(\beta) e^{-g_{1}(\beta) d} \times \\
& {\left[e^{-g_{2}(\beta) h}\left(g_{2}(\beta)-g_{0}(\beta)\right)\left(g_{2}(\beta)-g_{1}(\beta)\right)\right.} \\
& \left.-e^{g_{2}(\beta) h}\left(g_{2}(\beta)+g_{0}(\beta)\right)\left(g_{2}(\beta)+g_{1}(\beta)\right)\right]^{-1},
\end{aligned}
$$

with the radiation functions $g_{1}(\beta)=\sqrt{\beta^{2}-k_{0}^{2} \varepsilon_{r 1}}$ and $g_{2}(\beta)=\sqrt{\beta^{2}-k_{0}^{2} \varepsilon_{r 2}}$ evaluated as $g_{0}(\beta)$.

Then, we consider the homogeneous structure excited by a 2-D infinite along $z$ electric line source, located at $(X, Y)$ in region \#1. The Green's function is the electric field generated at $(x, y)$ by this line source. For the observation vector $(x, y)$ in region \#1, it is comprised of a primary (singular) term $G_{1}^{p r}$ and a secondary (smooth) term $G_{1}^{\text {sec }}$, respectively, given by

$$
\begin{aligned}
G_{1}^{p r}(x, y, X, Y) & =-\frac{j}{4} H_{0}^{(2)}\left(k_{0} \sqrt{(x-X)^{2}+(y-Y)^{2}}\right), \\
G_{1}^{s e c}(x, y, X, Y) & =\int_{-\infty}^{+\infty} \gamma_{1}^{s e c}(\beta) e^{g_{1}(\beta)(y+Y)} e^{-j \beta(x-X)} d \beta,
\end{aligned}
$$

where $H_{0}^{(2)}$ denotes the zeroth order and second kind cylindrical Hankel function, while

$$
\begin{aligned}
& \gamma_{1}^{\sec }(\beta)=e^{-2 g_{1}(\beta) d}\left[\cosh \left(g_{2}(\beta) h\right)\left(g_{1}(\beta)-g_{0}(\beta)\right) g_{2}(\beta)\right. \\
& \left.+\left(g_{0}(\beta) g_{1}(\beta)-g_{2}^{2}(\beta)\right) \sinh \left(g_{2}(\beta) h\right)\right] \times \\
& {\left[4 \pi g_{1}(\beta)\right]^{-1}\left[\cosh \left(g_{2}(\beta) h\right)\left(g_{1}(\beta)+g_{0}(\beta)\right) g_{2}(\beta)+\right.} \\
& \left.\left(g_{0}(\beta) g_{1}(\beta)+g_{2}^{2}(\beta)\right) \sinh \left(g_{2}(\beta) h\right)\right]^{-1} .
\end{aligned}
$$

For the observation point $(x, y)$ lying in region $\# 0$, the Green's function takes the form

$G_{0}(x, y, X, Y)=\int_{-\infty}^{+\infty} \gamma_{0}(\beta) e^{-g_{0}(\beta)(y-Y)} e^{-j \beta(x-X)} d \beta$, where

$$
\begin{aligned}
& \gamma_{0}(\beta)=\left[\pi g_{0}(\beta)\right]^{-1} e^{g_{0}(\beta)(d+h)-g_{1}(\beta) d} g_{0}(\beta) g_{2}(\beta) \times \\
& {\left[e^{g_{2}(\beta) h}\left(g_{0}(\beta)+g_{2}(\beta)\right)\left(g_{1}(\beta)+g_{2}(\beta)\right)\right.} \\
& \left.-e^{-g_{2}(\beta) h}\left(g_{0}(\beta)-g_{2}(\beta)\right)\left(g_{1}(\beta)-g_{2}(\beta)\right)\right]^{-1} .
\end{aligned}
$$

According to the fundamental analytical formula of the scattering integral (see e.g. p. 228 of [32]), the generated scattered electric field in the region \#1, due to the presence of the PEC cylinder, has the integral representation

$$
\begin{aligned}
E_{1, s c}(x, y) & =-j k_{0} \zeta_{0} \times \\
& \int_{C} K(l)\left[\begin{array}{c}
G_{1}^{p r}(x, y, X(l), Y(l)) \\
+G_{1}^{\text {sec }}(x, y, X(l), Y(l))
\end{array}\right] d l,
\end{aligned}
$$

where $K(l)$ is the axial current induced along the PEC cylinder's boundary $C$.

By taking into account the cylinder's small electrical size, we can make the so-called thin-wire approximation [28] and infer that the (unknown) current $K$ does not vary significantly around $C$ and, therefore, we may consider it as spatially constant. In this way, the scattered field $E_{1, s c}$ evaluated at the origin $(x, y)=(0,0)$ is approximated by (see also [28])

$$
\begin{aligned}
E_{1, s c}(0,0) \cong & -\frac{\pi k_{0} a \zeta_{0} K}{2} H_{0}^{(2)}\left(k_{0} a \sqrt{\varepsilon_{r 1}}\right) \\
& -2 \pi j k_{0} a \zeta_{0} K G_{1}^{s e c}(0,0,0,0) .
\end{aligned}
$$

By applying the PEC boundary condition for vanishing total electric field at the center of the PEC cylinder

$$
E_{1, b a c k}(0,0)+E_{1, s c}(0,0)=0,
$$

we obtain

$$
K=\frac{j}{2 \pi k_{0} a \zeta_{0}} \frac{E_{1, \text { back }}(0,0)}{\frac{j}{4} H_{0}^{(2)}\left(k_{0} a \sqrt{\varepsilon_{r 1}}\right)-G_{1}^{s e c}(0,0,0,0)} .
$$

Once the induced current $K$ is determined, we evaluate the scattered field in vacuum region $\# 0$ by using again the scattering integral as follows

$$
E_{0, s c}(x, y) \cong-2 \pi j k_{0} a \zeta_{0} K G_{0}(x, y, 0,0),
$$

which, by means of (3), takes the form

$$
E_{0, s c}(x, y) \cong-2 \pi j k_{0} a \zeta_{0} K \int_{-\infty}^{+\infty} \gamma_{0}(\beta) e^{-g_{0}(\beta) y-j \beta x} d \beta \text {. }
$$

The scattered power in the upper half-space is obtained by using the method of stationary phase [33], yielding

$$
P_{s c}=8 \pi^{4}\left(k_{0} a\right)^{2} k_{0} \zeta_{0}|K|^{2} \int_{0}^{\pi}\left|\gamma_{0}\left(k_{0} \cos \phi\right)\right|^{2} \sin ^{2} \phi d \phi,
$$

measured in Watt per meter of $z$-axis.

In our consideration, the host medium with permittivity $\varepsilon_{r 1}$ is the lossy ground, the metallic (PEC) inclusion represents the buried inclusion and the superstrate layer of permittivity $\varepsilon_{r 2}$ corresponds to a thin homogeneous layer deposited on the earth's surface. The applications related to buried objects in the ground usually utilize an operational UHF frequency $f$ in the range from $500 \mathrm{MHz}$ to $1 \mathrm{GHz}$. The variation of the 
complex dielectric permittivity $\varepsilon_{r 1}$ within the aforementioned frequency interval is negligible for all considered cases of very dry, medium dry or wet ground (similar considerations are made in [34]). In particular, the following frequency variations hold for each case (see e.g. [35]): (i) for very dry ground $\varepsilon_{r 1}^{d r y} \cong 3-j 0.05\left(\frac{f}{10^{6}}\right)^{-0.4} \cong 3-j 0.0036$, (ii) for medium dry ground: $\varepsilon_{r 1}^{\text {medium }} \cong 15-j 0.1\left(\frac{f}{10^{6}}\right)^{0.25} \cong 15-j 0.52$ and (iii) for wet ground: $\varepsilon_{r 1}^{w e t} \cong 30-j 3.2$, where $f$ is measured in units of $\mathrm{Hz}$.

Note that the complex ground permittivities have been approximated by their average value over the UHF frequency range indicated above. The validity of this approximation is demonstrated in Fig. 1(b) where the big markers (triangular, square and circular) correspond to the mean values and the small dots to the variations of the real and imaginary parts of $\varepsilon_{r 1}$ with respect to $f$ for $500 \mathrm{~Hz}<\frac{f}{10^{6}}<1000 \mathrm{~Hz}$. It is evident that the adopted mean values of $\varepsilon_{r 1}$ approximate quite accurately all values of $\varepsilon_{r 1}$ in the entire considered frequency range and hence we proceed in the sequel by using these mean values for each of the three considered cases of the ground. We note that it is possible to retain a frequency-dependence of $\varepsilon_{r 1}$ in the integral representation (4), however the difference is negligible since $\operatorname{Re}\left(\varepsilon_{\mathrm{r} 1}\right) \gg\left|\operatorname{Im}\left(\varepsilon_{\mathrm{r} 1}\right)\right|$ and the presentation of the results of Section III below, will become less intuitive.

\section{PARTICULARIZATION AND APPROXIMATION}

The basic aim of the present study is to find the physical properties (permittivity $\varepsilon_{r 2}$ ) and size (thickness $h$ ) of the deposited superstrate atop the ground which aid the detection of the buried (into depth $d$ ) PEC cylinder of radius $a$ from measurements of its response due to the moving scanning source (current $I$, distance $\chi$ ) excitation. In other words, we are searching for a layer that can "unlock" the ground in the sense that the presence of this specifically selected superstrate will make the two-layered configuration of the earth together with the superstrate to have effective properties which will increase the scattered power due to the the hidden target and hence reveal its presence to an external observer.

Obviously, the practical significance of the work would have been very limited if we confined our analysis to an inclusion of specific location and size; therefore, the proposed deposited layer should work for any type of inclusion inside the ground by substantially amplifying its scattered field. Such an amplification does not necessarily require an active medium; it can be achieved by a suitable redistribution of the fields into the superstrate layer and the half space. The layer should enhance the transmission of the incident illumination into the ground in order for the PEC cylinder to get maximally excited and at the same time it should not block the scattered field from the inclusion so that it transmits back to free-space. If one considers reciprocal superstrate (which is the case here), these two objectives are equivalent.

In order to find layers that perform this interplay between reflected and transmitted fields for the incident and the scattered components, we consider the inclusion-free structure excited in the simplest possible way: by a normally incident plane wave. The first assumption (inclusion-free) is made in order to obtain a solution that can work for every inclusion regardless of its position or size. The second assumption (plane wave incidence) is made for the sake of simplicity and in order to obtain results independent from the position of the line source. In Fig. 2(a), we depict the two cases of our simplified configuration: our aim is to maximize the transmission coefficient $T_{1}$ of the externally incident field into the earth and the transmission coefficient $T_{0}$ of the reflected field back to free space. These two are proportional to each other (due to reciprocity) and are defined as follows

$$
\begin{aligned}
& \frac{T_{0}}{\sqrt{\varepsilon_{r 1}}}=T_{1} e^{-j k_{0} h} \\
& =4 \sqrt{\varepsilon_{r 2}}\left[e^{j k_{0} h \sqrt{\varepsilon_{r 2}}}\left(1+\sqrt{\varepsilon_{r 2}}\right)\left(\sqrt{\varepsilon_{r 1}}+\sqrt{\varepsilon_{r 2}}\right)\right. \\
& \left.-e^{-j k_{0} h \sqrt{\varepsilon_{r 2}}}\left(1-\sqrt{\varepsilon_{r 2}}\right)\left(\sqrt{\varepsilon_{r 1}}-\sqrt{\varepsilon_{r 2}}\right)\right]^{-1} \equiv T .
\end{aligned}
$$
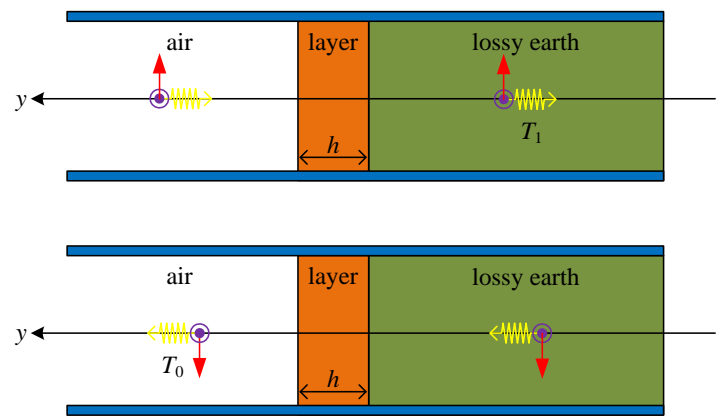

(a)

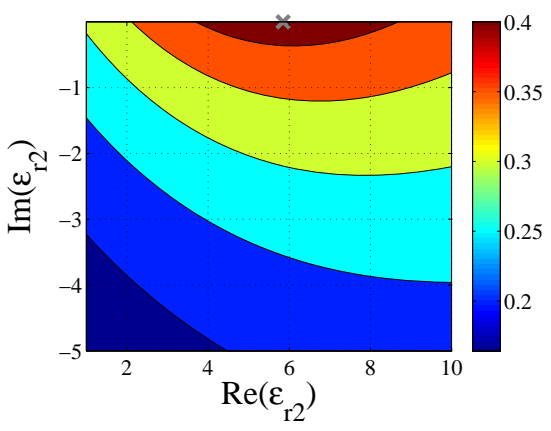

(b)

Fig. 2. (a) The physical configuration of the inclusion-free structure excited by normally incident plane waves used for obtaining optimal solutions in an approximate way. (b) Typical variation of the transmission magnitude $|T|$ on the complex $\varepsilon_{r 2}$ plane regardless of the ground permittivity $\varepsilon_{r 1}$. The gray "X" marks maximal $|T|$. Note that it corresponds to real $\varepsilon_{r 2}$.

The proportionality constant $\sqrt{\varepsilon_{r 1}}$ corresponds to the ratio of wave impedances between vacuum and host ground. For a fixed frequency $f$ (fixed wavenumber $k_{0}$ ) and a fixed type of ground $\varepsilon_{r 1}$, the quantity $|T|$ to be maximized is a sole function of a complex variable $\varepsilon_{r 2}$ and a positive real variable $k_{0} h$. In Fig. 2(b), we show a typical variation of function $|T|$ on the complex plane of the layer's permittivity $\varepsilon_{r 2}$ for a representative set of parameters (similar behavior is exhibited regardless of the permittivity $\varepsilon_{r 1}$ ). One can observe that the 
maximal value of $|T|$ (indicated by a gray " $\times$ ”) is achieved for a lossless superstrate $\left(\operatorname{Im}\left(\varepsilon_{r 2}\right)=0\right)$. That property cannot be considered to hold a priori for general layered systems but for this specific configuration and for the considered ranges of the structure's parameters it has been checked to be indeed valid. Therefore, we can restrict the parametric space by adopting only real permittivities $\varepsilon_{r 2}$. Additionally, we avoid exotic metamaterials or $\varepsilon$-near-zero (ENZ) materials, and hence consider that $\varepsilon_{r 2}>1$.

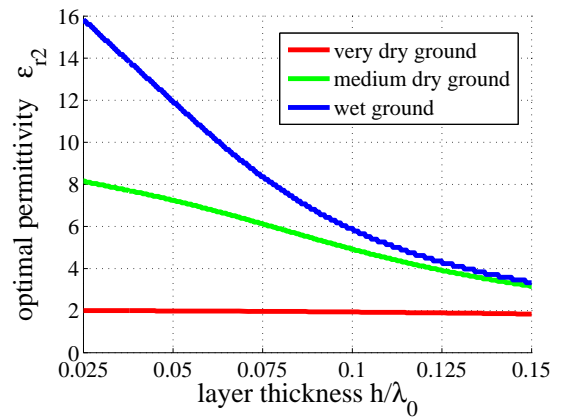

(a)

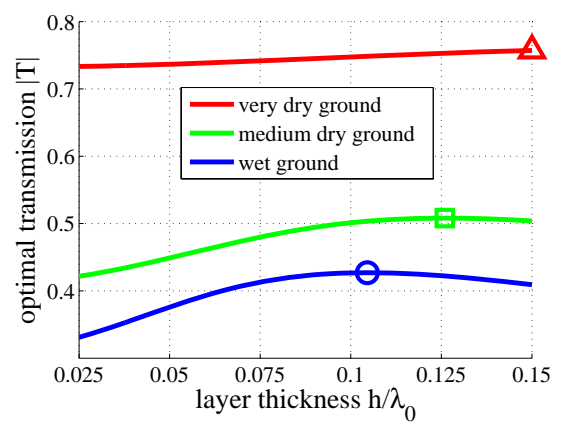

(b)

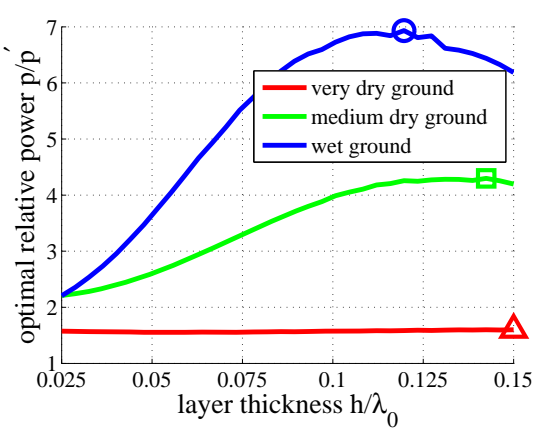

(c)

Fig. 3. (a) The optimal (maximal $|T|$ criterion) permittivity $\varepsilon_{r 2}$ of the deposited layer as function of the its electrical thickness $h / \lambda_{0}$ for various types of ground (inclusion-free configurations). (b) The maximum transmission magnitude $|T|=\left|T_{1}\right|=\left|T_{0}\right| /\left|\sqrt{\varepsilon_{r 1}}\right|$ as function of the its electrical thickness $h / \lambda_{0}$ for various types of ground (inclusion-free configurations). (c) The ratio of the scattering power integrals $p / p^{\prime}$ as function of the its electrical thickness $h / \lambda_{0}$ for various types of ground $\left(d=3 \lambda_{0}, a=0.1 \lambda_{0}\right)$.

Based on the latter assumptions, we can simplify our problem by maximizing a function $|T|$ of two positive variables: $\frac{h}{\lambda_{0}}>0$ and $\varepsilon_{r 2}>1$. We perform such a maximization operation for every single value of layer's electrical thickness $\frac{h}{\lambda_{0}}$ within a certain range and in Fig. 3(a), we show the optimal values of the permittivity $\varepsilon_{r 2}$ for the three sorts of ground $\left(\varepsilon_{r 1}\right.$ very dry, medium dry and wet). In other words, we depict all the combinations of electrical sizes $\frac{h}{\lambda_{0}}$ and dielectric constants $\varepsilon_{r 2}$ which lead to maximal transmission $|T|$ for each of the three ground type scenarios. Note the stability of the optimal material with respect to $\frac{h}{\lambda_{0}}$ for the case of a very dry ground and the convergence of the bestcase permittivities for the other two types of ground as $\frac{h}{\lambda_{0}}$ gets larger. Furthermore, the larger is the dielectric constant $\varepsilon_{r 1}$ of the ground, the larger is the optimal dielectric constant $\varepsilon_{r 2}$ of the layer. From these "optimal pairs", one should choose the one that gives the best transmission; such a selection is made in Fig. 3(b). Obviously, the optimal transmission is smaller the more lossy is the ground since the power lost during the travel and converted into heat becomes more substantial. The markers show the maxima of the three curves (one for each type) and indicate the electrical thicknesses $\frac{h}{\lambda_{0}}$ which, combined with the respective $\varepsilon_{r 2}$ given by Fig. 3(a), give the optimal solutions for the deposited layer for each of the three considered scenarios (very dry, medium dry, and wet ground).

The maximal transmissions of Fig. 3(b) and the indicated three optimal parameters for the layer $\left(k_{0} h_{\text {opt }}, \varepsilon_{r 2, \text { opt }}\right)$ correspond, according to our strategy described above, to the inclusion-free configuration. In order to test the performance of the determined optimal superstrates when the buried inclusion is present, we go back to the complete structure of Fig. 1(a) and the formula of the scattered power (10). A meaningful indicator of how much more detectable the inclusion becomes in the presence of the layer is the ratio of a superposition of the scattered powers for all possible positions of the line source. In particular, we define the following two integrals corresponding to scattered powers in the presence and in the absence $\left(\varepsilon_{r 2}=1\right)$ of the deposited layer, respectively

$$
p=\int_{-\mathcal{X}}^{+\mathcal{X}} P_{s c}(\chi) d \chi \quad, \quad p^{\prime}=\int_{-\mathcal{X}}^{+\mathcal{X}} P_{s c}^{\prime}(\chi) d \chi
$$

where $\mathcal{X}$ is a suitably large distance such that the evaluated integral values are stabilized. In Fig. 3(c), we show the ratios $\frac{p}{p^{\prime}}$ (for each type of ground) as functions of $\frac{h}{\lambda_{0}}$ for the permittivities indicated by Fig. 3(a). It is emphasized that (especially in the cases of medium dry and wet ground) the ratio of the scattered power integrals is substantial which verifies that the superstrate layers, selected through the analysis of the inclusion-free structure of Fig. 2(a), are indeed effective in amplifying the scattering response from the inclusion. Even more importantly, the variations of $\frac{p}{p^{\prime}}$ are quite stable with respect to $\frac{h}{\lambda_{0}}$ and the maxima of the curves are exhibited at points close to the corresponding ones that the curves of $|T|$ in Fig. 3(b) are maximized.

Therefore, it is verified that the strategy of simplifying the configuration (inclusion-free) and the excitation (plane wave) in order to extract the optimal dielectric constants $\varepsilon_{r 2, o p t}$ and electric thicknesses $k_{0} h_{o p t}$ is effective and yields satisfying results for the full problem of Fig. 1(a). For this reason, we are going to use the obtained solutions from this simplified problem in the following numerical results. 


\section{NumeriCAl Results}

\section{A. Arbitrary Materials}

The optimal dielectric constants $\varepsilon_{r 2}$, corresponding to the results of Fig. 3(b) referring to the simplified model, are the following:

(i) $\varepsilon_{r 2, o p t}^{d r y}=1.84$ for very dry ground,

(ii) $\varepsilon_{r 2, \text { opt }}^{\text {medium }}=3.88$ for medium dry ground,

(iii) $\varepsilon_{r 2, o p t}^{\text {wet }}=5.52$ for wet ground,

while the corresponding electrical thicknesses $k_{0} h$ of the deposited layer are:

(i) $k_{0} h_{\text {opt }}^{\text {dry }}=0.94$ for very dry ground,

(ii) $k_{0} h_{\text {opt }}^{\text {medium }}=0.79$ for medium dry ground,

(iii) $k_{0} h_{\text {opt }}^{\text {wet }}=0.66$ for wet ground.

As referred above, these optimal parameter values are derived by using the maximal $|T|$ criterion (independent from depth or shape of the inclusion); the criterion of maximal $\frac{p}{p^{\prime}}$ is mainly used for verification.

Figs. 4(a)-6(c) depict the variations of the scattered fields and their powers for the optimal aforementioned superstrate's parameters classified according to the considered type of ground. More precisely, first the very dry ground case is considered. Fig. 4(a) depicts the variations of the scattered power $P_{s c}$ versus the horizontal position $\chi$ of the primary line source when no superstrate is present and when the configuration is covered by the optimal superstrate layer. Moreover, Figs. 4(b) and 4(c) show the contour plots of the magnitude of the scattered electric field $E_{0, s c}$ in the air region as functions of the electrical coordinates $\left(\frac{x}{\lambda_{0}}, \frac{y}{\lambda_{0}}\right)$ when no superstrate layer is present and when the superstrate layer is the optimal one, respectively. From the symmetry of the distributions, it is obvious that Figs. 4(b) and 4(c) correspond to central excitation $(\chi=0)$. Figs. 5(a)-5(c) and 6(a)-6(c) depict the corresponding plots with Figs. 4(a)-4(c) for the cases of medium dry and wet ground respectively. It is evident that, for each of the three considered cases with respect to the ground, the scattered power increases for almost all line source's positions $\chi$. The increase is (relatively) larger for the wet ground which is the case in which the ground possesses the largest losses. Similar conclusions hold for the plots of $\left|E_{0, s c}\right|$ : the beneficial influence of the superstrate layer is evident in the sense that the scattered field in the air region increases considerably with the addition of the optimal superstrate; the more substantial increase is again observed for the wet ground. It is also worth to note that the horizontal region with respect to $\frac{x}{\lambda_{0}}$ for which larger scattered field is obtained shrinks as one moves from the very dry to the medium dry and finally to the wet ground. Hence, the location of the buried inclusion becomes most evident for the wet ground.

\section{B. Practical Realization}

The aforementioned optimal sets of superstrate's parameters $\left(k_{0} h_{\text {opt }}, \varepsilon_{r 2, \text { opt }}\right)$ have been obtained from a procedure that did not take into account practical realization limitations and constraints. As far as the thicknesses $k_{0} h_{\text {opt }}$ are concerned, the actual implementation error is proportional to the sensitivity of the utilized length measurement device. Since we are talking

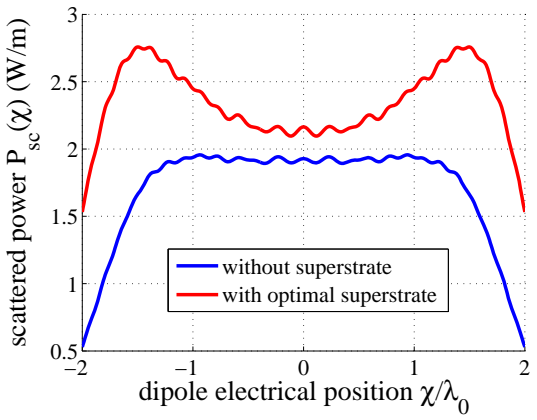

(a)

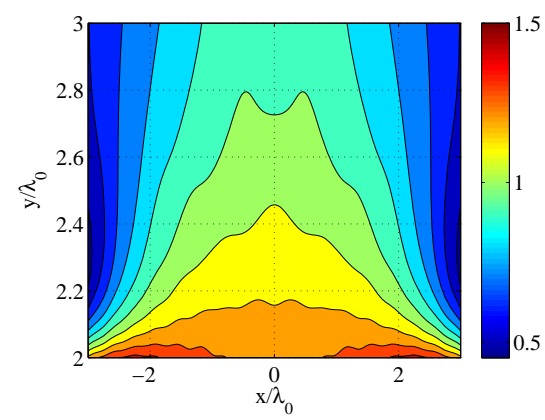

(b)

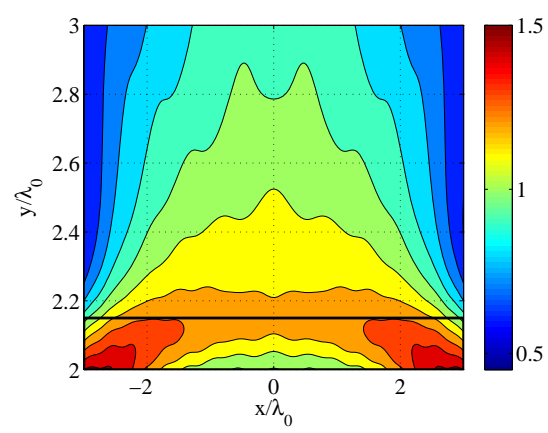

(c)

Fig. 4. (a) Scattered power $P_{s c}$ versus the position $\chi$ of the line source without any superstrate and with the optimal superstrate. (b) Contour plot of the magnitude of the scattered electric field $\left|E_{0, s c}(x, y)\right|$ in the air region on the map of the Cartesian electrical coordinates $\left(x / \lambda_{0}, y / \lambda_{0}\right)$ in the absence of the deposited layer $(\chi=0)$. (c) Contour plot of the magnitude $\left|E_{0, s c}(x, y)\right|$ on the map of $\left(x / \lambda_{0}, y / \lambda_{0}\right)$ in the presence of the optimal layer $(\chi=0)$. Plot parameters: very dry ground, $d=4 \lambda_{0}, a=\lambda_{0} / 5, f=750 \mathrm{MHz}$

about low radio frequencies, such an error is considered as negligible. However, it is difficult to fabricate a medium of specific dielectric constant $\varepsilon_{r 2, o p t}$ without suffering certain imperfections.

In this subsection, we are using specific natural/ordinary materials whose suitable mixtures can possess electromagnetic properties close to the ones dictated by the determined optimal permittivities. As a basic material we use a very common granular substance: sand, whose dielectric constant $\varepsilon_{r}^{\text {sand }} \cong 3$ does not have imaginary part and is dispersionless within the considered frequency range; see e.g. [36], [37], and [38, pp. 246-262]. By inspecting the permittivities $\varepsilon_{r 2, o p t}$ that we should effectively mimic, we need a sparser and a denser material than sand. For the sparser one, we can utilize 


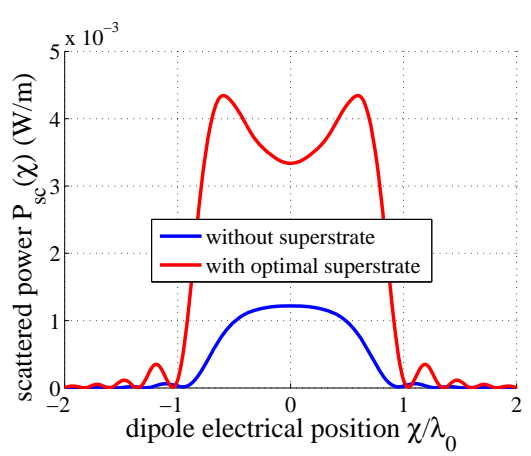

(a)

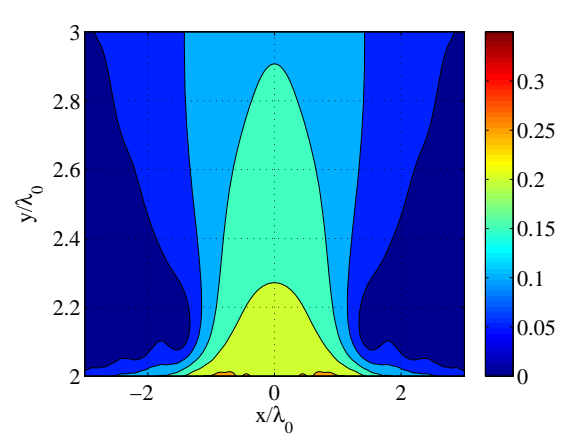

(b)

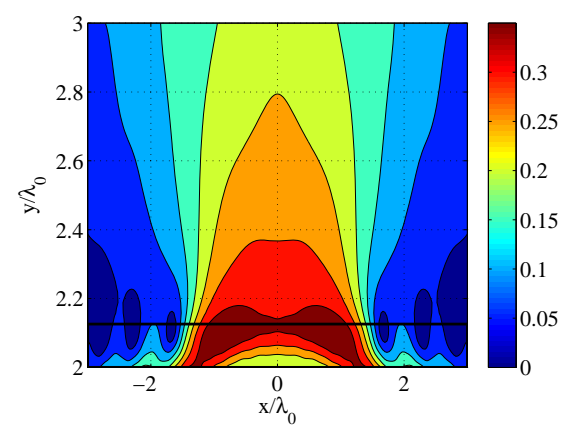

(c)

Fig. 5. The respective plots with those of Figs. 4(a)-4(c) for medium dry ground and $d=4 \lambda_{0}, a=\lambda_{0} / 5, f=750 \mathrm{MHz}$.

polyurethane foam whose properties are identical to those of vacuum $\varepsilon_{r}^{\text {foam }} \cong 1$ (at UHF band) and for a denser substance, we select 2-propanol which is the least lossy of the common available materials for low radio frequencies. The permittivity of propanol follows the following law [39]

$$
\varepsilon_{r}^{\text {propanol }} \cong 3.65+\frac{17}{1+0.00028 j\left(\frac{f}{10^{6}}\right)^{0.966}} .
$$

Our plan is to use sand as the host medium and proper inclusion of the two other substances (foam, propanol) so that the final mixture has effective permittivity similar to the optimal ones for each case. Certainly, there are many practical questions and difficulties to be answered in the operational use in the real world, like for example the deposition of the utilized materials on real ground. These issues can be mitigated by for example putting our mixture layer on top of a very thin sheet of inert and elastic polymer which guarantees the

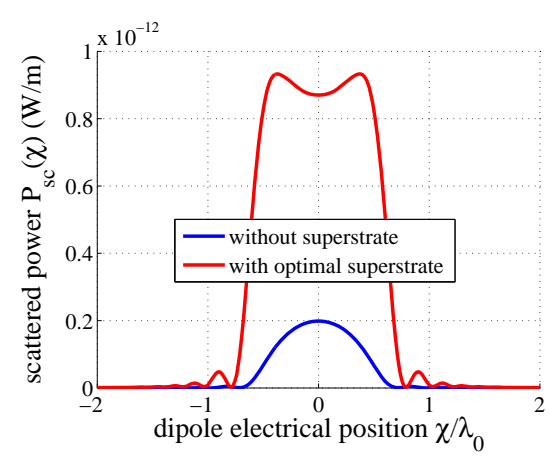

(a)

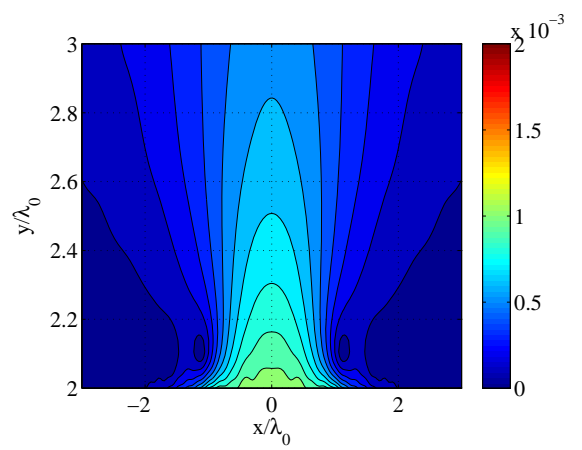

(b)

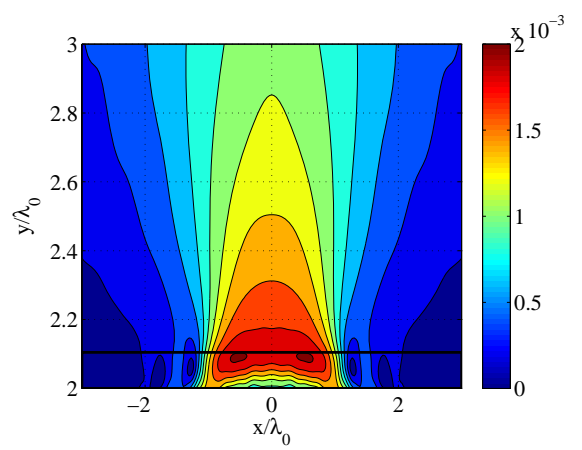

(c)

Fig. 6. The respective plots with those of Figs. 4(a)-4(c) for wet ground and $d=4 \lambda_{0}, a=\lambda_{0} / 5, f=750 \mathrm{MHz}$.

planar shape of the deposited layer. When it comes to the very dry ground scenario, the optimal effective dielectric constant $\varepsilon_{r 2, o p t}^{d r y} \cong 1.84$ can be directly obtained by mixing sand with foam and, in order to maximize the sparsifying effect of the foam, we use it in the form of small disks as indicated by the corresponding mixture formula. In particular, the permittivity of this substance (large number of polyurethane foam disks into sand) is given by [38, pp. 68]

$$
\begin{array}{r}
\varepsilon_{r 2}^{\text {dry }} \cong \varepsilon_{r}^{\text {sand }}+u_{d r y}\left(\varepsilon_{r}^{\text {foam }}-\varepsilon_{r}^{\text {sand }}\right) \times \\
\frac{2 \varepsilon_{r}^{\text {foam }}+\varepsilon_{r}^{\text {sand }}}{\left(3-u_{d r y}\right) \varepsilon_{r}^{\text {foam }}+u_{d r y} \varepsilon_{r}^{\text {sand }}},
\end{array}
$$

where $u_{d r y}$ is the volume filling factor of foam into sand. Note that both of the media used $\left(\varepsilon_{r}^{\text {sand }}\right.$ and $\left.\varepsilon_{r}^{\text {foam }}\right)$ are characterized by an absence of dispersion. Therefore we can 


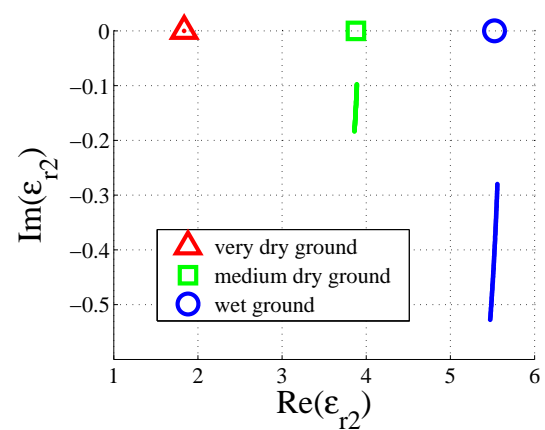

(a)

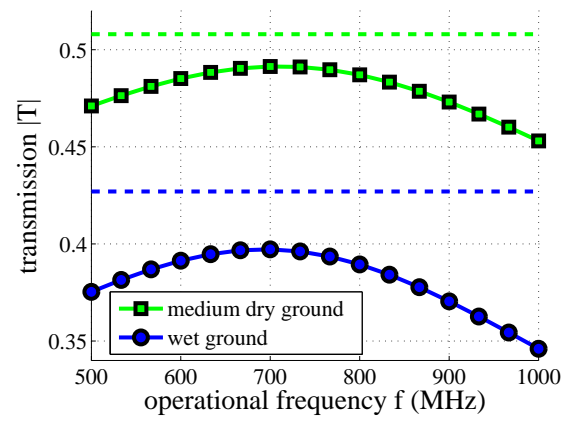

(b)

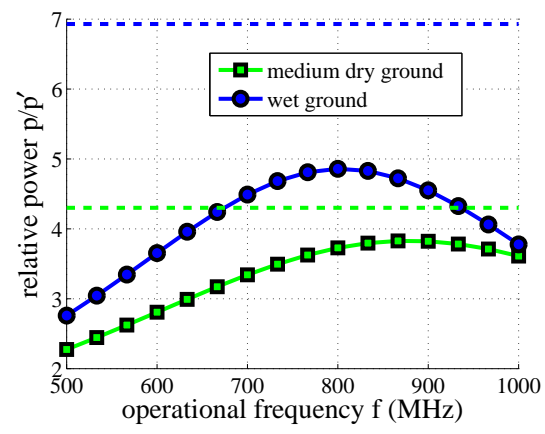

(c)

Fig. 7. (a) The real permittivities (mixture of sand and foam disks and mixture of sand and propanol, namely propanol-soaked sand) compared to the optimal ones. (b) The actual transmission magnitude. (c) The actual relative power. Dashed lines correspond to ideal results.

simply compute the proper filling factor by

$$
\operatorname{Re}\left(\varepsilon_{r 2}^{d r y}\right)=\varepsilon_{r 2, o p t}^{d r y} \Rightarrow u_{d r y} \cong 0.45 .
$$

It is stressed that almost half the volume of the obtained medium should be filled by small disks of foam something which is quite challenging in terms of homogenization. That situation would be even harder if we used other shapes of foam inclusions with weaker effect [38].

As far as the cases of medium dry and wet ground are concerned, the optimal effective dielectric constants $\varepsilon_{r 2, \text { opt }}^{\text {medium }} \cong$ 3.88 and $\varepsilon_{r 2, o p t}^{w e t} \cong 5.52$ can be directly obtained by mixing sand with 2-propanol (the procedure is quite straightforward, however manpower in form of mechanical assistants may be needed) and since the latter is liquid, simple weighted averages of the permittivities are quite accurate for the dielectric constant of the propanol-soaked sand

$$
\begin{aligned}
\varepsilon_{r 2}^{\text {medium }}(f) & \cong\left(1-u_{\text {medium }}\right) \varepsilon_{r}^{\text {sand }}+u_{\text {medium }} \varepsilon_{r}^{\text {propanol }}(f), \\
\varepsilon_{r 2}^{\text {wet }}(f) & \cong\left(1-u_{\text {wet }}\right) \varepsilon_{r}^{\text {sand }}+u_{\text {wet }} \varepsilon_{r}^{\text {propanol }}(f),
\end{aligned}
$$

where $u_{\text {medium }}, u_{\text {wet }}$ are the corresponding filling factors for the two medium dry and wet ground. Since the relations (16), (17) are dispersive, we select to equalize the real part of the aforementioned permittiviities $\varepsilon_{r 2}^{\text {medium }}(f), \varepsilon_{r 2}^{\text {wet }}(f)$ with the optimal ones $\varepsilon_{r 2, \text { opt }}^{\text {medium }}, \varepsilon_{r 2, \text { opt }}^{\text {wet }}$ only at the central frequency $\bar{f}=750 \mathrm{MHz}$ of the considered spectral range. In particular, the proper coefficients $u_{\text {medium }}, u_{\text {wet }}$ and the corresponding dispersive functions of the effective permittivity for the utilized materials are evaluated as follows

$$
\begin{array}{r}
\operatorname{Re}\left(\varepsilon_{r 2}^{\text {medium }}(\bar{f})\right)=\varepsilon_{r 2, \text { opt }}^{\text {medium }} \Rightarrow u_{\text {medium }} \cong 0.05 \\
\Rightarrow \varepsilon_{r 2}^{\text {medium }}(f) \cong 3.03+\frac{0.87}{1+0.00028 j\left(\frac{f}{10^{6}}\right)^{0.966}}, \\
\operatorname{Re}\left(\varepsilon_{r 2}^{\text {wet }}(\bar{f})\right)=\varepsilon_{r 2, o p t}^{\text {wet }} \Rightarrow u_{\text {wet }} \cong 0.15 \\
\Rightarrow \varepsilon_{r 2}^{\text {wet }}(f) \cong 3.09+\frac{2.5}{1+0.00028 j\left(\frac{f}{10^{6}}\right)^{0.966}} .
\end{array}
$$

Note that the $u_{\text {medium }}, u_{\text {wet }}$ are quite small. If we used water instead of propanol, the respective filling factors would be even smaller (because $\varepsilon_{r}^{\text {water }} \cong 81$ ) which could jeopardize the isotropic nature of the mixture. Furthermore, water is more lossy that 2-propanol which would deteriorate the performance of the (ideally lossless) layer in terms of amplifying the scatterer's response.

In Fig. 7(a), we mark the (real) optimal lossless values of the permittivity $\varepsilon_{r 2}$ for the three ground types (large markers) and the dielectric permittivity values depending on frequency $f$ for $500 \mathrm{~Hz}<\frac{f}{10^{6}}<1000 \mathrm{~Hz}$ given by (18), (19) of the actual mixtures (series of small dots). In the case of very dry ground, the employed materials are lossless and non-dispersive; thus, the actual solution is identical to the ideal one (and hence we do not examine it further). For the other two cases (medium dry and wet ground), the real part is very close to what should be for the entire frequency spectrum, while the imaginary part is moderate and dependent on $f$. The good coincidence of the real parts is natural since the filling factors are selected by equalizing the real permittivities and the frequency range is relatively small; furthermore, the losses are larger in the wet-ground scenario since more (lossy) propanol is needed to approximate $\varepsilon_{r 2, o p t}^{\text {wet }}$.

At this point, let us quantify the effect of these imperfections on the performance indicators of the proposed structures. In Fig. 7(b), we show the magnitude of the transmission coefficient $|T|$ as function of the operational frequency $f$ for the two types of ground, when the actual materials are used. With dashed lines we show the best transmission score for the optimal permittivities in each case. The quantity $|T|$ possesses substantial values for the entire frequency spectrum which are close to the optimal values. It is remarkable that transmission is not maximized at the central frequency $\bar{f}$, for which the 
real part of the permittivity is exactly equal to $\varepsilon_{r 2, o p t}$, but close to it. This happens due to the fact that the real part of the permittivity does not change substantially with frequency, contrary to the imaginary part. In other words, at a frequency $f=700 \mathrm{MHz}$, smaller than $\bar{f}$, the real part of the effective dielectric constant may be very close to the optimal value and the losses more diminished leading to higher transmissions. To test the proposed realizable structures, we evaluate also the criterion referred to the complete configuration (in the presence of the buried inclusion), namely the ratio $\frac{p}{p^{\prime}}$, as function of the operational frequency $f$. The results are plotted in Fig. 7(c). One directly observes that the magnitude of the represented quantity is quite high for both scenarios of the ground. The difference from the optimal performance (indicated by dashed lines) is larger for the wet ground than in the case of medium dry ground. Furthermore, the maxima of the curves are exhibited at frequencies higher than $\bar{f}$ which show the influence of the peripheral parameters (inclusion's depth and point nature of the source) on the full problem.

In Figs. 8, we show the integral of the scattered power over all positions of the source, defined in (12), normalized by the corresponding quantity $p_{0}$ when the inclusion is located at the ground surface $(d=0)$, as function of the electrical depth of the inclusion $\frac{d}{\lambda_{0}}$. Obviously, due to the presence of losses, the curves are downward sloping indicating that the detection of the inclusion is more difficult, the deeper is buried. Furthermore, the rate of decrease is smaller, the less lossy is the ground which is again attributed to the smaller attenuation the waves are subjected to. The graph of Fig. 8 has been produced for the optimal frequency $f=\bar{f}$ but, due to the normalization constant $p_{0}$ which varies with $f$, the graphs are very similar (almost identical) for every frequency within our operational range (for the same type of ground).

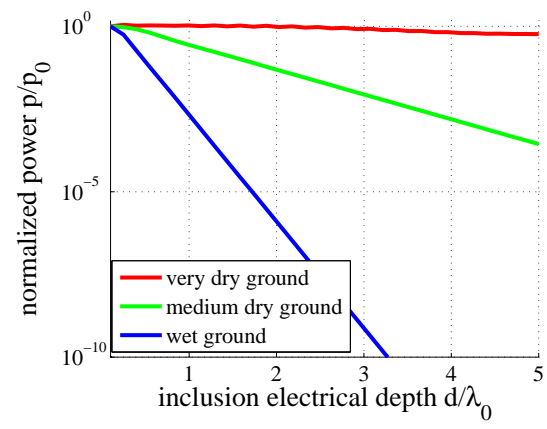

Fig. 8. The normalized integral of scattered power $p / p_{0}$ as function of the electrical depth of the inclusion $d / \lambda_{0}$ for several scenarios of the ground the central frequency: $f=\bar{f}=750 \mathrm{MHz}$. Plot parameter: $a=0.2 \lambda_{0}$.

\section{Additional Test Cases and Discussions}

We examine the validity and the performance of the developed methodology in some additional test cases. More specifically, we consider the scattering problem of a normally incident plane wave impinging on a buried inclusion of arbitrary shape at $d=1.8 \lambda_{0}$. Scattered field simulations are performed with COMSOL Multiphysics [40]; the considera- tion of the plane incident wave was selected for simplicity of the computations via COMSOL.

Figs. 9(a)-9(c) depict the magnitude of the scattered electric field (namely the difference between the total electric field and the background electric field; the latter is induced in the inclusion-free configuration composed of a three-layered dielectric medium) at all domains. It should be stressed that the the field in the interior of the inclusion is non-zero because we represent only the scattered component which should be of equal magnitude and opposite with the background one (or better its analytic continuation in the area of the object) to give a zero outcome at the cross section of the scatterer as imposed by the PEC nature of the buried inclusion. We consider planewave normal illumination for the case of a wet ground and of operating frequency $f=750 \mathrm{MHz}$. Figs. 9(a), 9(b) and 9(c) correspond, respectively, to an absent superstrate layer, to an optimal ideal superstrate and to an optimal realizable superstrate, where the ideal parameters are computed by the techniques of Sections II and III, while the realizable parameters by the procedure of Section IVB. The significant increase in the scattered field's values when using an optimal ideal layer is evident. Importantly, this increase remains still significant when using a realizable layer which approximates the parameters of the ideal layer. Moreover, it is worth to note that although the optimal parameters of the superstrate layer were calculated via the analysis of the boundary value problem of Section II, involving a line-source primary field and an inclusion of circular shape, these parameters may also offer a significant increase in the scattered field in the problem considered in this section corresponding to plane wave incidence on a buried inclusion of arbitrary shape.

Next, we examine the variations in the achieved scattered field's increase with respect to the changes of the operating frequency. To this end, we depict in Figs. 10(a) and 10(b) the magnitude of the scattered electric field at all domains at $f=750 \mathrm{MHz}$ for a medium dry ground and (a) an absent superstrate and (b) an optimal ideal superstrate. Figs. 10(c) and $10(\mathrm{~d})$ depict the magnitude of the scattered electric field at $f=500 \mathrm{MHz}$ and $f=1 \mathrm{GHz}$, respectively, where the parameters of the optimal superstrate layer are those computed for the same scattering problem at $f=750 \mathrm{MHz}$. It is observed that the superstrate's parameters determined to achieve enhanced scattered field's values at $f=750 \mathrm{MHz}$ also produce a substantial enhancement at $f=500 \mathrm{MHz}$ and $f=1 \mathrm{GHz}$.

\section{Concluding Remarks, Applicability Aspects AND FUTURE EXTENSIONS}

The traditional problem of detecting a buried inclusion into lossy earth was considered. In this study, we did not make any changes in the measuring process but we did change the configuration of the problem. In particular, we deposited a passive superstrate on the surface of the earth to redistribute the fields into all the regions (air, superstrate, ground) so that the transmission of the incident illumination is maximized. Using rigorous integral equation formulation, we showed that the scattered power due to the inclusion is significantly enhanced when an optimal superstrate layer is used and, importantly, 


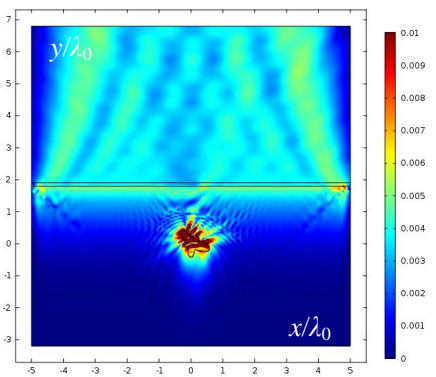

(a)

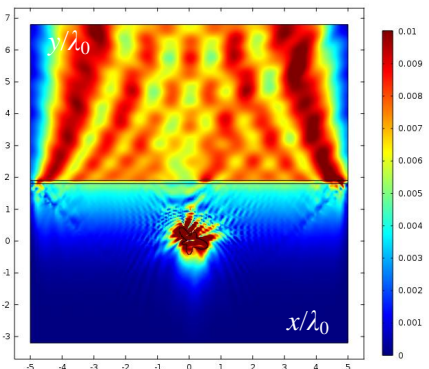

(b)

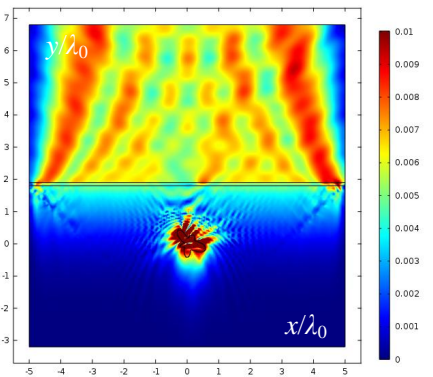

(c)

Fig. 9. Magnitude of the scattered electric field at all domains for $f=$ $750 \mathrm{MHz}$ and a wet ground and (a) an absent, (b) an optimal ideal, and (c) an optimal realizable superstrate. The buried inclusion has an arbitrary shape and is located at $d=1.8 \lambda_{0}$. Normally incident plane-wave illumination.

this power enhancement is not severely downgraded when conventional realizable materials are employed.

The implementation of the proposed method is expected to be quite inexpensive because realizing the permittivity values of the optimum superstrate layer resulting by using mixing formulas and mixing sand/water/propanol (according to the discussions of Section IVB) does not require the use of any costly materials. Moreover, since the superstrate layer needs only to be of centimeters to ten centimeters thick, the amount of the utilized materials is also reasonable. If very large areas are needed to be probed with the proposed method then the experimental procedure might become time-consuming. Still, this cost is in a way only a very "low-level" expense, requiring no skilled workforce or technical expertise; just mixing and subsequently deploying very inexpensive materials. In general, the labor needed for the production, transportation, and deposition of the mixture superstrate is a "low-cost" labor and certainly does not require technologically efficient realizations.

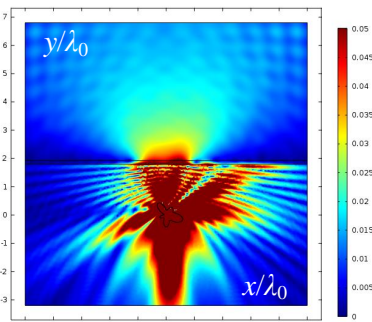

(a)

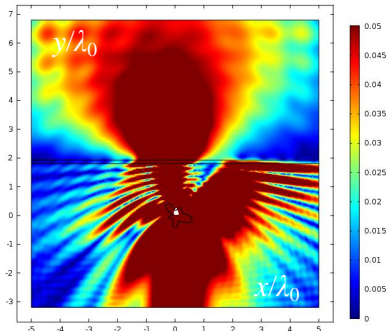

(c)

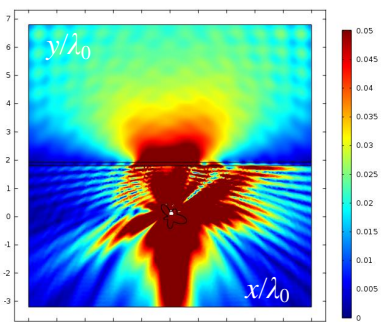

(b)

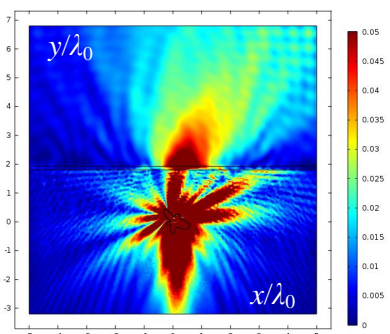

(d)
Fig. 10. Magnitude of the scattered electric field at all domains for a medium dry ground and (a) an absent superstrate at $f=750 \mathrm{MHz}$, and an optimal ideal superstrate at (b) $f=750 \mathrm{MHz}$, (c) $f=500 \mathrm{MHz}$, and (d) $f=1 \mathrm{GHz}$. The buried inclusion has an arbitrary shape and is located at $d=1.8 \lambda_{0}$. Normally incident plane-wave illumination.

An other issue is the fact that the determined parameters of the optimal superstrate depend in general on the variations of the ground's parameters. However, it is expected that the values of the superstrate's parameters obtained by the developed method are not so sensitive in variations of the other quantities of the problem (in the sense that when a characteristic of the configuration varies then beneficial influence in the scattered field is still observed). Partial evidence of this fact was already reported in Section IVC above where it was shown that a substantial change in the operating frequency does not at all deteriorate the achieved field enhancement. In any case, if in an experimental implementation it is anticipated that the ground's characteristics are variable, then the optimal superstrate's parameters can be easily controllable by changing the mixing ratios of the utilized two-component mixture.

The proposed method is non-invasive, and thus can be very useful in many applications where non-destructive remote sensing methods are required. Hence, our approach would be perfectly suited to those applications where one cannot or may not mechanically go below the surface of the ground, i.e. cannot dig in (or even if one could dig, that would be much slower than an electromagnetic scanning method). Some indicative applications to this direction are all kinds of civilian applications of GPR's, like nondestructive testing of structures and pavements, locating utility lines or buried structures, tunnels etc. Other areas could be archaeological searches and mapping as well as environmental applications like characterizing the pollution state of ground or even perhaps for gold nugget prospecting in riverbeds. Particularly, concerning the detection of land mines or UXO's, some special precautions need to be taken in the practical implementation 
of the proposed approach. For example, some type of remote control of setting the optimized layer on top of sites, where there is an indication that land mines or UXO's may be located, could prove useful. Moreover, environmental consequences after the utilized material is removed should be taken into account in cases that the ground is to be subsequently exploited. For example, if the ground surface is on a farming field and it is of importance not to jeopardize the continuation of cultivation then the effect of the material mixtures on the ground could be examined and in some cases other substances be sought. Nevertheless, in other applications, like archaeology which may take place in deserts, there would certainly be no harm and problems of deploying sand mixtures or polluting agricultural areas would perhaps not be so important. When it comes to demining applications, then there is of course a tradeoff: to win is more (discovering and getting rid of mines) than lose (environmental concerns due to the utilized mixture).

Several interesting future extensions of the presented method are feasible. A rough surface ground could be considered which would model properly bouldered terrains. However, since we are working at low microwave frequencies, the surface roughness can be in most cases safely considered quite small so that the assumption of a flat ground surface can accurately approximate realistic situations. Indeed, for a moderately rough ground surface the relative rms surface height deviation is small compared to the operating wavelength. Moreover, non-PEC buried inclusions could be tested in order to find how much the "unlocking" effect is deteriorated compared to the PEC case. Is is expected that the method would work with similar accuracy and also produce a considerable amplification of the scattered wave. This is due to that our approach is based on the inclusion-free structure and, as shown in details in Section III above, the superstrate layers, selected through the analysis of the inclusion-free structure, are indeed very effective in amplifying the scattering response from the inclusion. The 2-D solution, obtained in this work, is expected to be quite accurate in realistic applications when the intention is to try to detect elongated objects which can be approximated as 2-D in the transverse plane; in such a case some a priori knowledge of the direction the long axis of the object is pointing is required. The extension of the method to 3-D scattering is complicated but feasible. The dyadic Green's function has to be considered and subsequently expanded into Fourier integrals involving the vector eigenfunctions in the corresponding coordinate system (e.g. spherical or spheroidal system; in the spherical one these are the vector spherical wave functions $\mathbf{M}$ and $\mathbf{N}$ ). For an electrically small scatterer the mean value theorem for vector triple integrals can be invoked in order to extend the procedure leading to the derivation of the approximate expression (9) of the present 2-D analysis.

Other future research steps would be to test how the achieved enhancement in the scattering response of the buried inclusion is translated into improvement of the detecting inclusion accuracy (related to position, size and texture) of the standard methods. It is also interesting to test how the addition of a superstrate affects the measuring and the algorithmic procedures of inverse scattering when secondary obstacles are present and how the overall detection performance changes.

\section{REFERENCES}

[1] A. C. Dubey, and R. L. Barnard (Eds.), Detection and remediation technologies for mines and minelike targets, Proc. SPIE Int. Soc. Opt. Eng. 3079, 1997.

[2] J. M. Bourgeois, and G. S. Smith, "A complete electromagnetic simulation of the separated-aperture sensor for detecting buried land mines," IEEE Trans. Antennas Propag., vol. 46, pp. 1419-1426, 1998.

[3] S. Constable and L. J. Srnka, "An introduction to marine controlledsource electromagnetic methods for hydrocarbon exploration," Geophysics, vol. 72, no. 2, pp. WA3WA12, Mar.-April 2007.

[4] S. D. Khan and S. Jacobson, "Remote sensing and geochemistry for detecting hydrocarbon microseepages," Geological Soc. Am. Bulletin, vol. 120; no. 1/2, pp. 96-105, Jan.-Feb. 2008

[5] H. Huang and I. J. Won, "Automated anomaly picking from broadband electromagnetic data in UXO survey," Geophysics, vol. 68, pp. 18701876, 2003.

[6] H. Huang, B. SanFilipo, A. Oren, and I. J. Won, "Coaxial coil towed EMI sensor array for UXO detection and characterization," J. Applied Geophysics, vol. 61, pp. 217-226, 2007.

[7] L. Jr. Peters, J. J. Daniels, and J. D. Young, "Ground penetrating radar as a subsurface environmentals sensing tool," Proc. IEEE, vol. 82, pp. 1802 $1822,1994$.

[8] C. Liu, and L. C. Shen, "Numerical simulation of subsurface radar for detecting buried pipes," IEEE Trans. Geosci. Remote Sens., vol. 29, pp. 795-798, 1991.

[9] Y. C. Lin and K. Sarabandi, "Electromagnetic scattering model for a tree trunk above a tilted ground plane," IEEE Trans. Geosci. Remote Sens., vol. 33, pp. 1063-1070, 1995.

[10] J. He, T. Yu, N. Geng, L. Carin, "Method of moments analysis of electromagnetic scattering from a general three-dimensional dielectric target embedded in a multilayered medium," Radio Science, vol. 35, no. 2, pp. 305-313, Mar.-Apr. 2000.

[11] D. J. Daniels, Subsurface Penetrating Radar, IEE Press, ch 1, 1996.

[12] L. Crocco and F. Soldovieri, "GPR prospecting in a layered medium via microwave tomography," Annals of Geophysics, vol. 46, no. 3, pp. 559572, June 2003.

[13] T. J. Cui, W. C. Chew, A. A. Aydiner, and S. Chen, "Inverse Scattering of Two-Dimensional Dielectric Objects Buried in a Lossy Earth Using the Distorted Born Iterative Method," IEEE Trans. Geosc. Rem. Sens., vol. 39, no. 2, pp. 339-346, Feb. 2001.

[14] T. J. Cui, W. C. Chew, A. A. Aydiner, and Y. H. Zhang, "Fast-Forward Solvers for the Low-Frequency Detection of Buried Dielectric Objects," IEEE Trans. Geosc. Rem. Sens., vol. 41, no. 9, pp. 2026-2036, Sep. 2003.

[15] L.-P. Song and Q. H. Liu, "Fast three-dimensional electromagnetic nonlinear inversion in layered media with a novel scattering approximation," Inverse Problems, vol. 20, pp. S171-S194, 2004.

[16] L.-P. Song, Q. H. Liu, F. Li, and Z. Q. Zhang, "Reconstruction of ThreeDimensional Objects in Layered Media: Numerical Experiments," IEEE Trans. Antennas Propagat., vol. 53, no. 4, pp. 1556-1561, Apr. 2005.

[17] A. Savin, A. Bruma, R. Grimberg, S. Leitoiu, R. Steigmann, and C. C. Comisu, "Increasing the Probability of Detection and Evaluation of Buried Metallic Objects by Data Fusion GPR-Low Frequency Electromagnetic Sensor Array," Proceedings of 4th European-American Workshop on Reliability of Non-Destructive Evaluation, 2009.

[18] C. Yu, M. Yuan, Y. Zhang, J. Stang, R. T. George, G. A. Ybarra, W. T. Joines, and Q. H. Liu, "Microwave Imaging in Layered Media: 3-D Image Reconstruction From Experimental Data," IEEE Trans. Antennas Propagat., vol. 58, no. 2, pp. 440-448, Feb. 2010.

[19] T. B. Hansen and P. M. Johansen, "Inversion Scheme for Ground Penetrating Radar That Takes into Account the Planar Air-Soil Interface," IEEE Trans. Geosc. Rem. Sens., vol. 38, no. 1, pp. 496-506, Jan. 2000.

[20] P. Meincke, "Linear GPR Inversion for Lossy Soil and a Planar Air-Soil Interface," IEEE Trans. Geosc. Rem. Sens., vol. 39, no. 12, pp. 2713 2721, Dec. 2001.

[21] H. Brunzell, "Detection of Shallowly Buried Objects Using Impulse Radar," IEEE Trans. Geosc. Rem. Sens., vol. 37, no. 2, pp. 875-886, Mar. 1999.

[22] W. Al-Nuaimy, Y. Huang, M. Nakhkash, M. T. C. Fang, V. T. Nguyen, and A. Eriksen, "Automatic detection of buried utilities and solid objects with GPR using neural networks and pattern recognition," $J$. Applied Geophysics, vol. 43, pp. 157-165, 2000.

[23] D. Potin, P. Vanheeghe, E. Duflos, and M. Davy, "An Abrupt Change Detection Algorithm for Buried Landmines Localization," IEEE Trans. Geosc. Rem. Sens., vol. 44, no. 2, pp. 260-272, Feb. 2006. 
[24] E. Pasolli, F. Melgani, and M. Donelli, "Automatic Analysis of GPR Images: A Pattern-Recognition Approach," IEEE Trans. Geosc. Rem. Sens., vol. 47, no. 7, pp. 2206-2217, Jul. 2009.

[25] P. C. Chaumet, K. Belkebir, and R. Lencrerot, "Three-dimensional optical imaging in layered media," Optics Express, vol. 14, no. 8, pp. 3415-3426, Apr. 2006.

[26] C. A. Valagiannopoulos and N. K. Uzunoglu, "Simplified model for EM inverse scattering by longitudinal subterranean inhomogeneities exploiting the dawn/dusk ionospheric ridge", IET Microw. Antennas Propagat., vol. 5, no. 11, pp. 1319-1327, Aug. 2011.

[27] C. A. Valagiannopoulos and N. K. Uzunoglu, "Scattering of ELF waves by underground formations because of night-day ionospheric ridge", Radio Science, vol. 42, doi: 10.1029/2007RS003682, 2007.

[28] C. A. Valagiannopoulos, "Closed-form solution to the scattering of a skew strip field by metallic pin in a slab", Progress Electrom. Research, vol. 79, pp. 1-21, 2008.

[29] J. Jenwatanavet and J. T. Johnson, "An Analytical Model for Studies of Soil Modification Effects on Ground Penetrating Radar," IEEE Trans. Antennas Propagat., vol. 49, no. 6, pp. 923-933, Jun. 2001.

[30] R. Linnehan and C. Rappaport, "Mitigating Ground Clutter Effects for Mine Detection with Lightweight Artificial Dielectrics," Proceedings of SPIE, vol. 4742, pp. 259-268, 2002

[31] L. Capineri, F. Fiesoli, and C. Windsor, "Holographic radar: A strategy for uneven surfaces," 2012 14th International Conference on Ground Penetrating Radar (GPR), IEEE Catalog ISBN 978-1-4673-2660-5, proceedings of GPR2012, pp. 148-150.

[32] C.-T. Tai, Dyadic Green Functions in Electromagnetic Theory, IEEE Press, New York, 1994.

[33] C. A. Valagiannopoulos, N. L. Tsitsas, and A. H. Sihvola, "Hiding a bump on a PEC plane by using an isotropic lossless dielectric layer", IEEE Trans. Antennas Propagat., vol. 62, no. 11, pp. 5706-5714, Nov. 2014.

[34] C. A. Valagiannopoulos, "A signal coverage model for two neighboring islands of different size", Progress In Electromagnetics Research M, vol. 2, pp. 115-130, 2008.

[35] Electrical characteristics of the surface of the earth, Recommendation ITU-R P.527-3, 1992.

[36] United States Department of Agriculture, Soil Quality Indicators, USDA Natural Resources Conservation Service, Technical Report, 2008.

[37] United States Department of Agriculture, Soil Bulk Density Physical Properties, USDA Cooperative Soil Survey, Technical Report, 2015.

[38] A. H. Sihvola, Electromagnetic Mixing Formulas and Applications, IEEE Electromagnetic Waves Series, 1999.

[39] A. Boughriet, Z. Wu, H. McCann, and L. E. Davis, "The measurement of dielectric properties of liquids at microwave frequencies using open-ended coaxial probes", 1st World Congress on Industrial Process Tomography, pp. 318-322, Buxton, UK, April 14-17, 1999.

[40] Home Page of COMSOL Multiphysics. [Online]. Available: http://www.comsol.fi

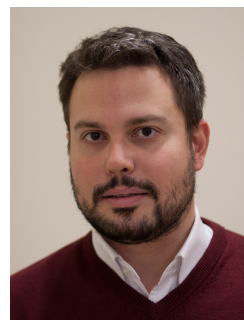

Constantinos A. Valagiannopoulos (M'12) was born in Athens, Greece in 1982. He received with honors the Dipl. Eng. degree in Electrical Engineering from National Technical University of Athens (NTUA), Greece in 2004, and the Ph.D. degree for studies on Electromagnetic Theory in 2009. From 2010 to 2015, he worked as a Postdoctoral Researcher in the Department of Radio Science and Engineering of the Aalto University, Finland. During the academic year 2014-2015, he was with the Laboratory of Metamaterials and Plasmonics in the Department of Electrical and Computer Engineering at the University of Texas at Austin, USA. In 2015, he joined Nazarbayev University (NU), Kazakhstan, as an Assistant Professor at Physics Department of the School of Science and Technology.

Dr. Valagiannopoulos's research interests include artificial metamaterials and their applications in devices controlling the electromagnetic fields. He has authored or coauthored over 65 works in international refereed scientific journals and presented numerous reports in international scientific conferences.

Dr. Valagiannopoulos is the winner of the inaugural 2015 JOPT research excellence award for his work: Perfect absorption in graphene multilayers. $\mathrm{He}$ was also the recipient of the International Chorafas Prize for the Best Doctoral Thesis in 2008 and the Academy of Finland Postdoctoral Grant for 2012-2015.

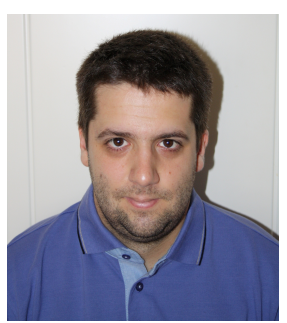

Nikolaos L. Tsitsas (S'03-M'06) was born in Athens, Greece, in 1979. He received the Diploma and Ph.D. degree in electrical engineering from the National Technical University of Athens (NTUA), Athens, Greece, in 2002 and 2006, respectively, and the M.Sc. degree in applied mathematics from the National and Kapodistrian University of Athens, Athens, Greece, in 2005

From 2008 to 2011, he was an Adjunct Lecturer at the School of Applied Mathematical and Physical Sciences of the NTUA. From 2009 to 2011, he was an Adjunct Lecturer at the Hellenic Army Academy. Since 2012, he has been an Assistant Professor at the Department of Informatics, Aristotle University of Thessaloniki. He is the author or coauthor of 42 papers in scientific journals and over 50 papers in conference proceedings. His research interests include analytical and numerical methods in direct and inverse wave scattering and propagation theory and applied mathematics.

Dr. Tsitsas is a member of the Optical Society of America, the American Mathematical Society, and the Technical Chamber of Greece.

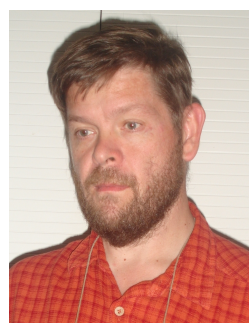

Ari H. Sihvola (S'80-M'86-SM'91-F'06) was born on October 6th, 1957, in Valkeala, Finland. He received the degrees of Diploma Engineer in 1981, Licentiate of Technology in 1984, and Doctor of Technology in 1987, all in Electrical Engineering, from the Helsinki University of Technology (TKK), Finland. Besides working for TKK and the Academy of Finland, he was visiting engineer in the Research Laboratory of Electronics of the Massachusetts Institute of Technology, Cambridge, in 1985-1986, and in 1990-1991, he worked as a visiting scientist at the Pennsylvania State University, State College. In 1996, he was visiting scientist at the Lund University, Sweden, and for the academic year 2000-01 he was visiting professor at the Electromagnetics and Acoustics Laboratory of the Swiss Federal Institute of Technology, Lausanne. In the Summer of 2008, he was visiting professor at the University of Paris XI, France.

Ari Sihvola is professor of electromagnetics in Aalto University School of Electrical Engineering (former name before 2010: Helsinki University of Technology) with interest in electromagnetic theory, complex media, materials modelling, remote sensing, and radar applications. He is Chairman of the Finnish National Committee of URSI (International Union of Radio Science), Chairman for the Commission B of International URSI, and Fellow of IEEE. 